\title{
Toward Conducting Motivational Interviewing with an On-Demand Clinician Avatar for Tailored Health Behavior Change Interventions
}

\author{
Christine L. Lisetti \\ and Ugan Yasavur \\ School of Computing and \\ Information Sciences \\ Florida International University \\ Miami, Florida 33199 \\ http://ascl.cis.fiu.edu
}

\author{
Ubbo Visser \\ Department of Computer Science \\ University of Miami \\ Miami, Florida, USA \\ web.cs.miami.edu/aigames/
}

\author{
Naphtali Rishe \\ School of Computing and \\ Information Sciences \\ Florida International University \\ Miami, Florida, USA \\ http://hpdrc.fiu.edu
}

\begin{abstract}
In this article we describe work-in-progress about the development of avatar-based personalized assistants that can delivered motivational interviewing health behavior change interventions, tailored to its specific users Our approach combines the latest progress in Embodied Conversational Agents (ECAs), believable agents, and dialog systems. We discuss how we use different platforms to aim at providing accessibility of personalized health assistant, anytime anywhere.
\end{abstract}

\section{INTRODUCTION}

We discuss our work-in-progress on the development of an intelligent Embodied Conversational Agent (ECA) or avatar that (1) generates multimedia, multilingual and multicultural specific tele-health messages, (2) tailors them to the diverse preferences of at-high-risk youth (e.g. gender, ethnicity, language), and (3) delivers them to youth seamlessly on personal computers, web-based systems, and/or mobile phones.

In particular, we are working on an intelligent agent architecture that integrates artificial intelligence techniques with various forms of multimedia technologies to connect at-risk youth to the healthcare system. The intelligent agent communicates with the at-risk youth via an interface with digital avatars or Embodied Conversational Agents (ECAs) [1], [2] who can engage the at-risk youth via innovative multilingual, multicultural, and multimodal messages promoting healthy behaviors.

To build on avatar acceptance, the health-promoting digital avatars are specifically tailored to the youth preferences (e.g. gender, ethnicity, language) and adaptable to the youth behaviors (e.g. specific risky behaviors). In addition, the need for dynamic and timely message delivery in the specific context of at-risk youth behaviors (sexual activity, alcohol/drug use) is being addressed by working on the design of avatars that youth can interact with it seamlessly depending upon the time of day, on a personal computer, on a web-based portal, or on a mobile smart phone [3], [4] (e.g. the iPhone, Android, are popular with most people and particularly with youth).
Latest findings have already shown that health promotion interventions tailored to the individual are an improvement over one-size-fits-all interventions [5]. Efficient individually tailored health promotion interventions became feasible with the advent of computer delivered interventions (CDI).

We posit that because ECAs can be designed to (1) be personalized to a user with a user-model, (2) be highly adaptive in terms of characteristics (e.g. gender, race, culture, language), (3) gather and remember feedback voluntarily given by a user, (4) have deep knowledge of a domain with specific expertsystems, and (5) be able to engage in meaningful dialog, they might be ideal to assist in delivering tailored health information to at-risk populations.

In addition, the possibility to run them on a variety of communication technologies (e.g. internet-based platforms, PCs) including mobile phones, so that their accessibility and availability improves, augments considerably their potential impact for at-risk populations, in a window of opportunity situation during which motivation to change may be especially malleable (e.g. when just arrested in a police station for substance use).

The concept of avatar-based interactive mobile interventions to promote health behavior has many applications in telemedicine and public health and promises to have major potential impact for future tele-health interventions that will go beyond the scope of the current applicative context such as interventions for drug and alcohol users, over-eaters, delivering tailored educational health material in a dynamic and personalized way.

Whereas the use of conversational agents online and on mobile devices has been a topic of on-going research over the past few years (see [6] for a review), the use of avatar-based system to help at risk-youth has not received much attention. Furthermore, the use of the recently developed motivational interviewing [7] counseling style for tailoring health behavior change interventions for at-risk and/or underserved populations has only started to be explored in the last couple of 
years [8], [9], [10], [11]

\section{Overall System COMPONEnTS}

We give a brief overview of the various components of our work-in-progress system, that implements on-demand clinician avatars capable of delivering motivational interviewing interventions that are tailored to address the needs of a specific user or patient.

\section{A. Motivational Interviewing}

Our personalized assistants deliver behavior change health interventions adapted from motivational interviewing (MI) [12], [7], [13]. MI is a directive, client-centered counseling style whose main goal is to magnify discrepancies that exist between client goals and current behavior. Rollnick and Miller [13] describe MI as helping clients to explore and resolve ambivalence [about reducing substance involvement], and enumerate the spirit of MI as:

1) Motivation to change is elicited from the client, and not imposed from without.

2) It is the clients task, not the counselors, to articulate and resolve his or her ambivalence.

3) Direct persuasion is not an effective method for resolving ambivalence.

4) The counseling style is generally quiet and eliciting.

5) The counselor is directive in helping the client to examine and resolve ambivalence.

6) Readiness to change is not a client trait, but a fluctuating product of interpersonal interaction.

7) The therapeutic relationship is more like a partnership or companionship than expert/recipient roles.

MI typically involves a brief assessment followed by feedback about the assessment results. Assessment data compiled from an individual are used to provide individualized, age- and gender-graded feedback about drinking and related behaviors. Throughout the MI interaction, a client-centered counseling style is used.

The inspiration of our avatar-based MI intervention described in [9] follows most closely the MI-related intervention developed for college students by Marlatt [14].

In our motivational interviewing intervention for students, participants are provided with personalized feedback as is done in many brief interventions [15].

Specifically, topics covered in the feedback session include personalized feedback in the areas of:

- self-reported drinking,

- perceived current and future risks of drinking,

- negative consequences of current consumption patterns,

- personal goals and their relation to alcohol use,

- social supports and their beliefs about drinking, and

- intentions and self-efficacy in regard to personal drinking goals.

We provide students with written, and verbal feedback about their own substance use vis--vis age- and gender-specific national norms. The belief that "everyone does it" is challenged via this feedback [16].

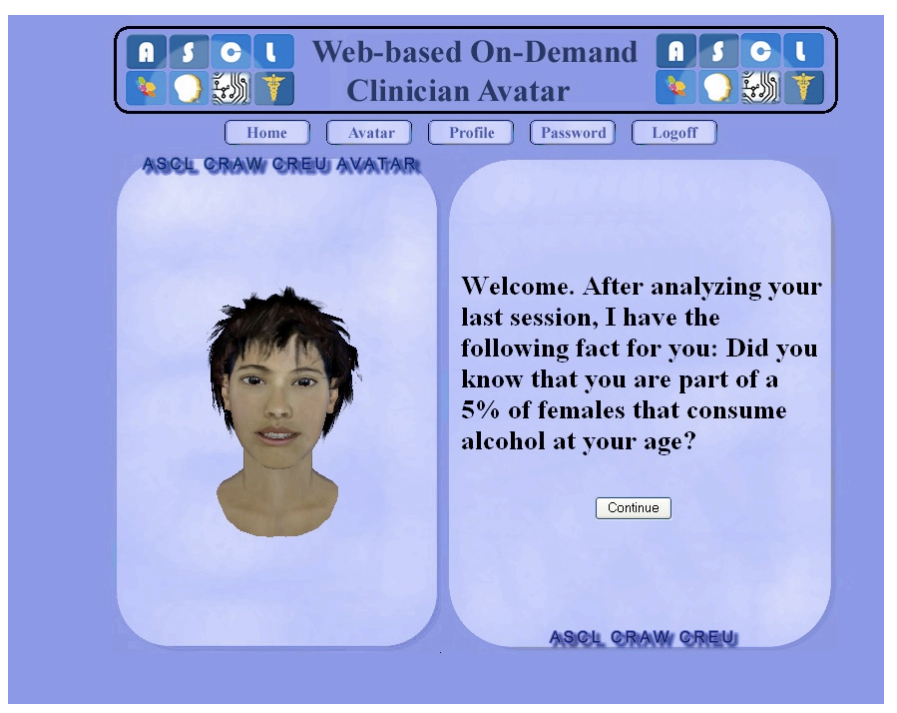

Fig. 1. Avatar provides tailored feedback

\section{B. Case Scenario}

The advantages of our avatar-based approach is to enable (relatively) easy authoring of intervention variations (as discussed in the components section).

Our current intervention is intended for use by students who qualify as drinkers (e.g. 6 or more drinking occasions in the past year). Once identified, drinkers are invited to participate in computerized health evaluation and feedback.

The On-Demand Avatar Clinician features a 3D emotionally expressive animated narrator who reads all material (such that no reading literacy is required). The repeatable intervention is conducted with the following primary MI components:

1) self-monitoring about drinking patterns and associated (problem behavior);

2) feedback regarding the negative consequences of alcohol use that the participant reported, as well as self- reported readiness to change and alcohol use as compared to that of same age and gender peers; these could be computerized and available as reminders;

3) pros and cons of alcohol use and related change, in which the participant chooses from lists of positive and negative aspects of alcohol use from their perspective. The benefits are identified during the interview, and maintained available as reminders by the system;

4) summary of overall exchange, with learned facts and current stage of change, is given and replayable;

5) question regarding the participants interest in change. Depending upon that answer, an optional goal-setting sub-session regarding alcohol use reduction is activated.

\section{System Components}

The system involves the following components.

A database stores data collected by social workers from college students on drug and alcohol use (e.g. age, gender, ethnicity, drug and drinking patterns, life goals). 


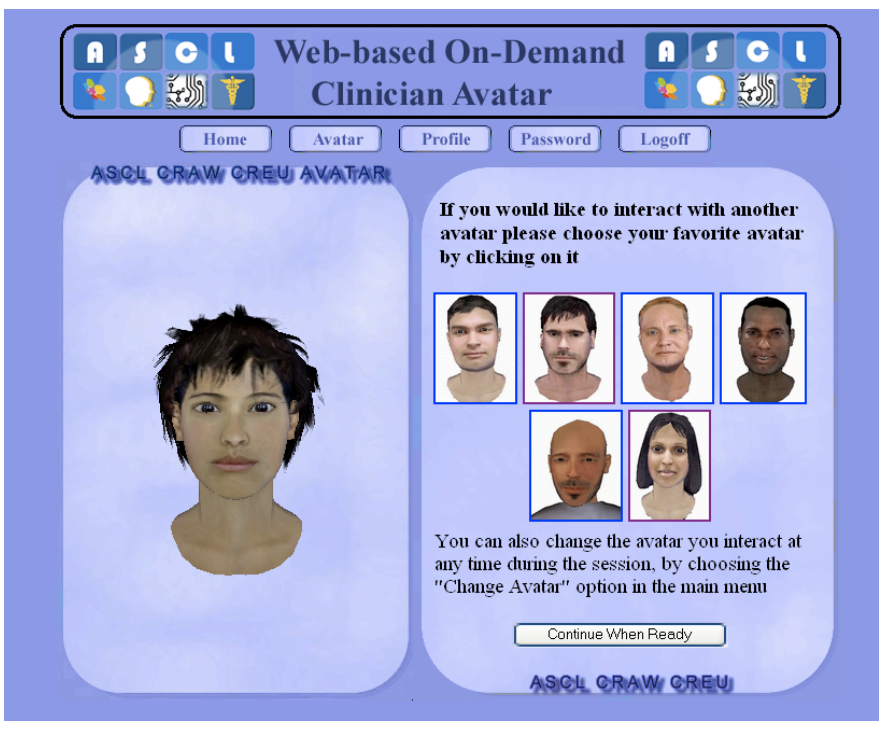

Fig. 2. Web Interface gives choice of avatars

The MI intervention is implemented with menu-based decision-tree navigation. Categories of sub-interventions are created for tailoring to specific students. For example, if the student is ready to move to a different stage in the wheel of change, then a sub-intervention appropriate for that stage is activated [17].

The normative statistical databases are accessed to calculate meaningful information about the student's specific drinking patterns as opposed to the norm (the belief that everyone does it is dispelled that way) (see Figure 1).

The User-Model combines Artificial Intelligence pattern recognition with high-level interpretation of a users habits and behaviors can be used to build a profile of a specific user. It includes the preferred users avatar identified either directly by the user or inferred by the system based on the users ethnicity (implementing patients' concordance) as shown in Figure 2.

The user-model also enables tailoring the intervention to that specific student user, by using the his or her name personalization, characteristics of the user such as gender adaptiveness, or self-identified needs of the user feedback-provision. Tailoring can also be dialog-based by asking the user about their goals and by recommending choices about how to best achieve these goals. The key is that the personalized, adaptive, or feedback based tailored messages are more effective in promoting attitude and behavior change than the generic one size fits all content delivered by print or to groups [18].

Accessible Web-Interface platform: The avatar and ECA architecture is integrated with a web-based interface which we prototyped so as to give as many desirable multilingual and multiethnic possible interactions.

Mobile Phone Platforms: New uses of mobile technologies indicate that mobile phones can be useful in the context of psychotherapy and counseling [3], [4]. Our individualized onDemand avatar clinician is a companion that can be available at all times on the portable device of young users at-risk
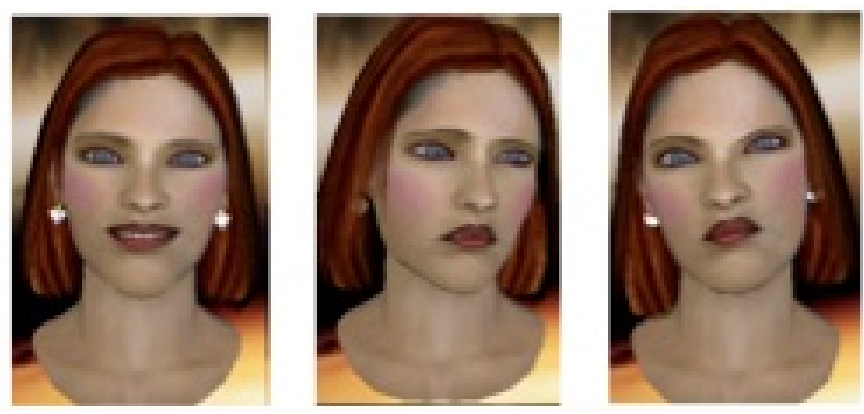

Fig. 3. Dynamic Facial Expression Animations

to provide them innovative multimedia support if and when needed. Although at a very experimental state, our current mobile avatar software package allows for both male and female avatars, different ethnicities, and universal facial expression animation [19].

\section{The Avatar Itself}

We have already identified specific features of ECAs that are important if not necessary to include in the design of social orthotic systems or computer-based interventions for health promotion behavior [20]. We integrate the following essential parts of the ECA into our prototype: 3-dimensional (3D) graphical avatar (facial and/or half/body) [21]; a set of features to portray different ethnicity features on the 3D avatar (e.g. skin color, facial proportions); a set of custom-made facial expressions capable of animating the 3D avatar with different facial expressions (e.g. pleased, sad, annoyed); textto-speech (TTS) engine to be able to read text with a natural voice; set of different natural languages to enable the avatar to be multilingual (English first, and spanish will follow); the possibility to manipulate vocal intonation in order to add to the agents believability; an interface allowing these features to be manipulated during the WOZ experiments and participatory design sessions.

Empathy: Empathy is considered the most important core condition in terms of promoting positive outcomes in psychotherapeutic contexts [22]. Considerable progress in animating virtual characters via mark-up language such that it is feasible to have text-to-speech scripted interventions [23]. We are interested in modeling some meaningful empathetic communication in the context of MI.

Our expressive avatar is programmed with believable facial expressions (see Figure3) developed in an earlier study [24] and vocal intonation synthesis to make believable and congruent with the dialog content during the interaction with the youth.

\section{CONCLUSION}

In this article we have described work-in-progress on the development of an on-demand avatar clinician to deliver and conduct motivational interviewing behavior change interventions. The demand to make health promotion interventions that are tailored to an individual's specific needs is real, and 
the technological advance that are describing is an attempt to address that need.

Our next step will be to program parts of the MI intervention using dialog-based engine and natural language processing.

Dialog Abilities and Spoken Language Generation: A number of studies have pointed out that one of the limits of computers for psychotherapy lies in their partial ability to adequately interpret natural language inputs (Plutchik and Karasu, 1991). Whereas latest progress in natural language processing in general and in social dialog abilities of ECAs in particular [25], have been increasing ECAs dialog abilities steadily, full natural language understanding is still not within reach. However, given the domain-specific nature of our avatar-based health interventions which follow semi pre-determined scripts, meaningful interventions can be designed without full natural language processing abilities. We are currently identifying the restricted specific dialog scripts that will be used by the ECA. Believability and Engagement:

\section{ACKNOWLEDGMENT}

The authors would like to thank Eric Wagner for introducing us to motivational interview and how it was used in the NIAAA-funded adolescent behaviors and lifestyle evaluation (ABLE) program.

\section{REFERENCES}

[1] J. Cassell and E. J. Sullivan, S. Prevost, \& E. Churchill, Embodied Conversational Agents. MIT Press, 2000.

[2] J. Cassell, T. Bickmore, M. Billinghurst, L. Campbell, K. Chang, H. Vilhjalmsson, and H. Yan, "Embodiment in conversational interfaces: Rea," in CHI '99: Proceedings of the SIGCHI conference on Human factors in computing systems. New York, NY, USA: ACM Press, 1999, pp. 520-527.

[3] C. Bang, M., Timpka, T., Eriksson, H., Holm, H., \& Nordin, "Mobile phone computing for in situ cognitive behavior therapy," in Proceedings of the World Congress on Medical Informatics (Medinfo), 2007.

[4] Y.-K. Lim and S.-D. K. Alexander Fedorov, "D- Caricature Generation System on the Mobile Handset Using a Single Photograph," in International Conference on Parallel Processing Workshops, 2007.

[5] G. R. Lustria ML, Cortese J, Noar SM, "Computer-tailored health interventions delivered over the Web: review and analysis of key components." Patient Education and Counselingi, vol. 74, no. 2, pp. 156-173, 2009.

[6] T. Bickmore and T. Giorgino, "Methodological Review : Health Dialog Systems for Patients and Consumers," Journal of Biomedical In39formatics, vol. 39, no. 5, pp. 556-571, 2006.

[7] S. E. Miller, W. R. \& Rollnick, Motivational interviewing: 2nd Ed. Preparing people to change addictive behavior, S. E. Miller, W. R. \& Rollnick, Ed. New York: Guilford Press., 1991.

[8] C. L. Lisetti, "Embodied Conversational Agents for Psychotherapy," in CHI 2008 workshop on mental health.

[9] C. L. Lisetti and E. Wagner, "Mental health promotion with animated characters: Exploring issues and potential," in AAAI Spring Symposium, 2008. [Online]. Available: http://www.aaai.org/Papers/Symposia/Spring/2008/SS08-04/SS08-04-013.pdf

[10] D. Schulman, T. Bickmore, and C. Sidner, "An Intelligent Conversational Agent for Promoting Long-term Health Behavior Change using Motivational Interviewing," in 2011 AAAI Spring Symposium Series, 2011, pp. 61-64. [Online]. Available: http://www.aaai.org/ocs/index.php/SSS/SSS11/paper/download/2401/2854

[11] B. Magerko, J. Dean, A. Idnani, M. Pantalon, and G. D'Onofrio, "Dr. Vicky: A Virtual Coach for Learning Brief Negotiated Interview Techniques for Treating Emergency Room Patients," in AAAI Spring Symposium, 2011
[12] G. S. Miller, William R.; Rose, "Toward a theory of motivational interviewing," American Psychologist, vol. 64, no. 6, pp. 527-537, 2009.

[13] W. Rollnick S, \& Miller, "What is motivational interviewing?" Behavioural and Cognitive Psychotherapy, vol. 23, pp. 325-334., 1995. [Online]. Available: http://www.stephenrollnick.com/index.php/allcommentary/64-what-is-motivational-interviewing

[14] E. Marlatt, G. A., Baer, J. S., Kivlahan, D. R., Dimeff, L. A., Larimer, M. E., Quigley, L. A., Somers, J. M., \& Williams, "Screening and brief intervention for high-risk college student drinkers: Results from a 2-year follow-up assessment," Journal of Consulting \& Clinical Psychology, vol. 66, pp. 604-615, 1998.

[15] T. M. DiClemente, C. C., Bellino, L. E., \& Neavins, "Motivation for change and alcoholism treatment," Alcohol Research \& Health, vol. 23 , pp. 86-92, 1999.

[16] G. Dimeff, L.A., Baer, J.S., Kivlahan, D.R., \& Marlatt, Brief Alcohol Screening and Intervention for College Students (BASICS): A harm reduction approach. New York: Guilford, 1999.

[17] J. O. Prochaska and W. F. Velicer, "The transtheoretical model of health behavior change." American journal of health promotion AJHP, vol. 12, no. 1, pp. 38-48, 1997. [Online]. Available: http://www.ncbi.nlm.nih.gov/pubmed/10170434

[18] V. Strecher, "Computer-tailored smoking cessation materials: a review and discussion," Patient Education Counseling, vol. 36, no. 2, pp. 107$117,1999$.

[19] P. Ekman, "The Argument and Evidence about Universals in Facial Expressions of Emotion," in Handbook of Social Psychophysiology, H. Wagner and A. Manstead, Eds. Chichester, England, Wiley, 1989, pp. 143-164.

[20] C. L. Lisetti, "Features for culturally appropriate avatars for behaviorchange promotion in at-risk populations," Studies In Health Technology And Informatics, vol. 144, no. 22-26, 2009. [Online]. Available: www.ncbi.nlm.nih.gov

[21] U. Visser and C. L. Lisetti, "Avatars in a Modern Soccer Manager," in Proceedings of the ARDE workshop at AAMAS, 2010.

[22] M. Bellet, P. and Maloney, "The importance of empathy as an interviewing skill in medicine." Journal of the American Medical Association; vol. 266, pp. 1831-1832, 1991

[23] H. Predinger and M. Ishizuka, Life-Like Characters. Springer, NY, 2004.

[24] M. Paleari, A. Grizard, and C. L. Lisetti, "Adapting Psychologically Grounded Facial Emotional Expressions to Different Anthropomorphic Embodiment Platforms," in Proceedings of the FLAIRS Conference 2007, 2007.

[25] T. Bickmore and J. Cassell, "Negotiated Collusion: Modeling Social Language and its Relationship Effects in Intelligent Agents." User Modeling and Adaptive Interfaces, vol. 13, pp. 89-113, 2003. 